\title{
Photochemical bonding of special optical fibers
}

\author{
Sławomir Ertman ${ }^{1}$, Monika Bielska ${ }^{1}$, Aleksandra Czapla ${ }^{1}, \mathrm{Krzysztof} \mathrm{Domosławski}^{1}$, Miłosz Chychłowski ${ }^{1}$, \\ Karolina Reszka ${ }^{1}$, and Tomasz R. Woliński ${ }^{1}$
}

${ }^{I}$ Faculty of Physics, Warsaw University of Technology, Koszykowa 75, 00-662 Warszawa,

Received September 24, 2014; accepted September 30, 2014; published September 30, 2014

\begin{abstract}
The most popular method for permanent connecting of optical fibers is thermal bonding with fusion or filament splicers. However, this method in some cases is not suitable, for example if the connected fibers are made of materials with different melting temperatures (i.e. silica and soft glass fibers) or in the case of microstructured optical fibers filled with materials which are highly sensitive to high temperature (i.e. organic materials such liquid crystals). In this work we will present some practical issues of an alternative method of permanent connecting various types of optical fibers by gluing with the use of photo-curable adhesives.
\end{abstract}

Almost every device (i.e. sensor or all-in-fiber component) that is based on a special optical fiber (SOF) requires permanent connections between such a fiber and other types of optical fibers. There are many fibers that can be considered as "special", i.e. higly-birefringent fibers, nonlinear optical fibers, tunable optical fibers, fibers made of non-silica glasses or the whole range of microstructured optical fibers. Generally speaking, that term can cover all optical fibers which guiding or physical properties differ from so-called "standard" or "classic" optical fibers [1]. If the SOF is all solid and based on silica glass, the connections with other silica fibers can be made with fusion or filament splicers [2, 3]. Also microstructured optical fibers based on silica glass can be effectively spliced, although in most cases some local collapse of microholes is observed [4]. The situation becomes more complicated if two fibers made of different materials have to be connected, especially if the melting temperature of one fiber is much lower than the other [5] (i.e. soft or multi-component glasses, polymers in comparison to silica glass). Also microstructured fibers filled with various substances are not suitable for thermal splicing, in particular if the substance could be damaged or even destroyed with a high temperature (i.e. organic materials, including liquid crystals). In a situation in which fibers cannot be connected with a thermal method, the main alternative is to use direct fiber gluing. Such a method has been already used to create devices based on liquid crystal filled microstructured fibers [6], as well as for connecting silica and fluoride fibers [7, 8]. In this work we report our progress in permanent connecting of various types of optical fibers with the use of photocurable adhesives. We will also discuss some practical issues which appear in photochemical bonding, in particular positioning connected fibers and some techniques of application of small volumes of adhesives.

The idea of photochemical optical fibers is quite simple and intuitive. First, the fibers have to be cleaved and cleaned at the ends. Then the fibers have to be aligned and a desirable amount of a photo-curable adhesive should be placed at the interface between fibers. Usually, after the application of glue the fiber alignment has to be corrected to optimize the coupling. Finally, the bond has to be cured with an adequate light source (usually UV light).

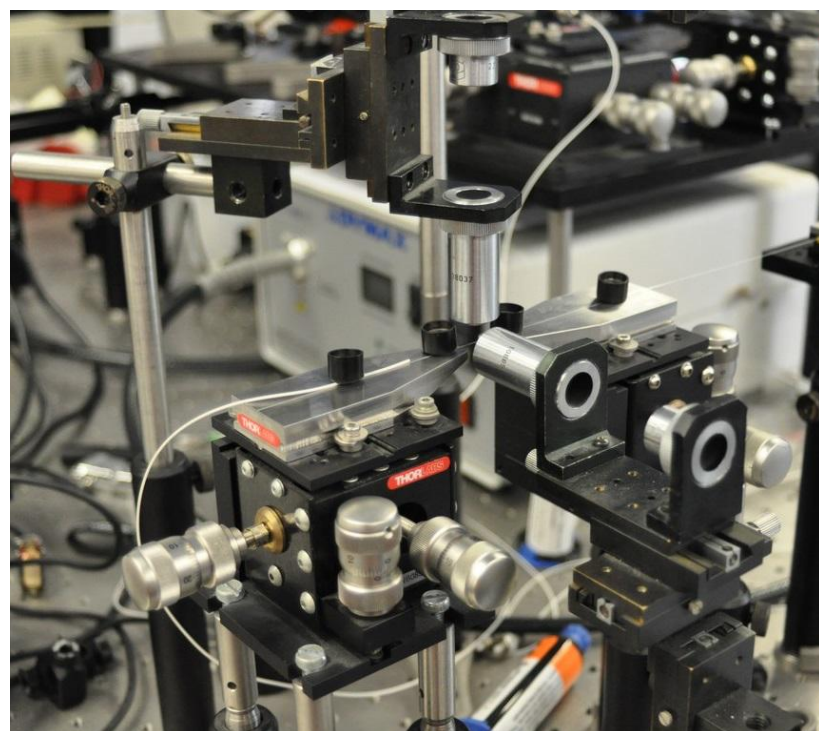

Fig. 1. In the first approach we used 3-axis positioning, provisory microscopes and manual application of the adhesive - the results was not always acceptable and improvement of the process was needed.

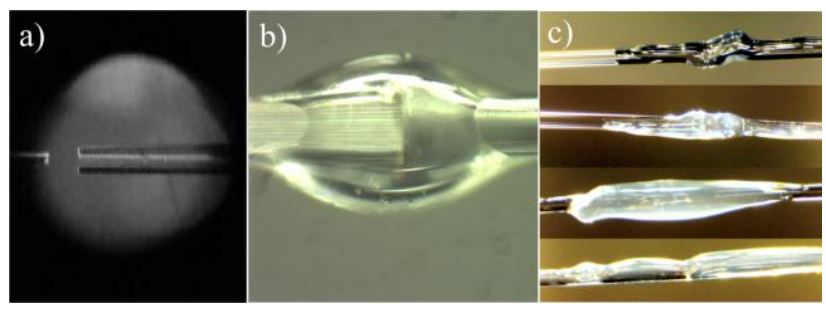

Fig. 2. Typical view from the provisory microscope assemblies shown above (a) and example of a good connection between PCF an SMF ,but with quite large droplet (b) and examples of non-symmetrical connections made with manual application of the adhesive (c). 
In the first approach we used 3-axis positioning of the fibers which alignment was visually controlled with selfmade microscope setups (Fig. 1). Such visual control was not very effective (it was not possible to observe the fibers from both sides and the viewing area was limited - Fig 2a). In this setup the application of an adhesive was made manually, usually by using additional fiber with a drop of glue at the end. It was possible to obtain a good quality of connections between various types of fibers (i.e. Fig. 2b) but in most cases the bond created in such a way was quite non-symmetrical and sometimes even breakable (Fig. 2c).

In order to make the gluing procedure more efficient and convenient we made a few improvements of the setup. The 3-axis positioning was replaced with a 5-axis alignment stage, allowing not only xyz-movement but also angular adjustment of the fibers (it appeared that in most cases angular adjustment significantly improved coupling efficiency). To improve the quality of visual observation we replaced self-made microscopes with digital microscopes so it was possible to observe the fibers simultaneously from both sides on the computer screen (Fig. 3). Finally, instead of unrepeatable manual application of glue, we decided to use an applicator mounted on a mechanical arm, which position was precisely controlled in a 3-axis.
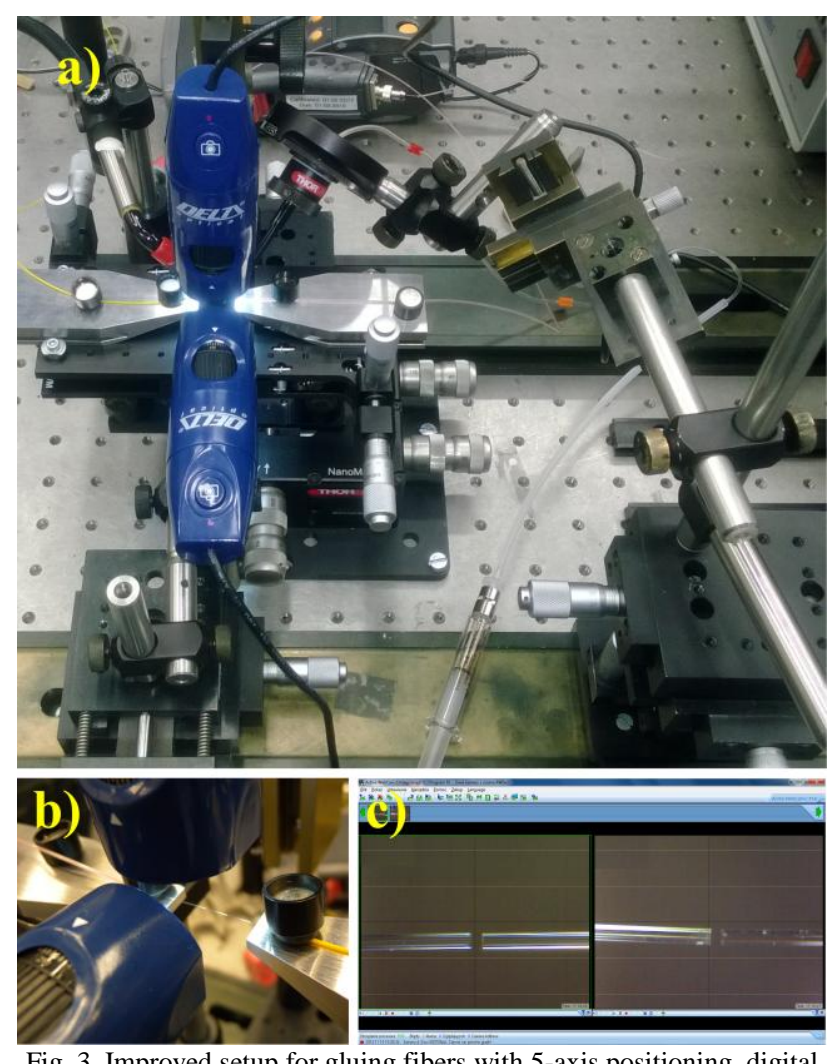

Fig. 3. Improved setup for gluing fibers with 5-axis positioning, digital view control from both sides and precisely controlled glue applicator: a) overall view of the setup; b) close-up of the connected fibers; c) screenshot with simultaneous pictures from both cameras 5-axis positioning and digital view control significantly improved the quality of alignment (not only in terms of coupling efficiency but also in the context of time needed for fibers alignment). But the most important improvement was the possibility to control glue application precisely. We have tested two different methods of adhesive application. In the first method a drop of glue was placed at the end of the medical needle, which diameter was a few times larger than that of the connected fibers (to ensure that most of the drop will remain on the needle). When the end of the needle was in contact with the fibers and then moved away, only a small amount of the adhesive was left on the fibers' surface (Fig. 4a). It was also possible to move the needle precisely along the fibers and in such a case a thin layer of glue remained on the surface of the fibers (Fig. 4b). We have tested such a method of application for many types of adhesives, with various viscosities and the effects were always similar - only a small volume of glue was left and small bonds could be created. We also noticed that the mechanical properties were good even for very small bonds. However, it was very important to cure the glue with a high power UV lamp. We have tested many types of adhesives, ie. Drei Bond (6023, 6020, 6022, 6060), Loxeal $(3022,3023)$ or Dymax (OP-29, OP-29-Gel, OP24-Rev-B, OP-4-20632, OP-4-20632-GEL, OP-67-LS, $425,429)$ and for each glue it was possible to obtain good curing with few seconds by using Dymax Blue-Wave 75 UV lamp. Some adhesives could be also cured with violet lasers $(405 \mathrm{~nm})$ but, in general, we advise to use a lamp (much shorter time of curing and our impression is that the bonds cured with a lamp have better mechanical properties).

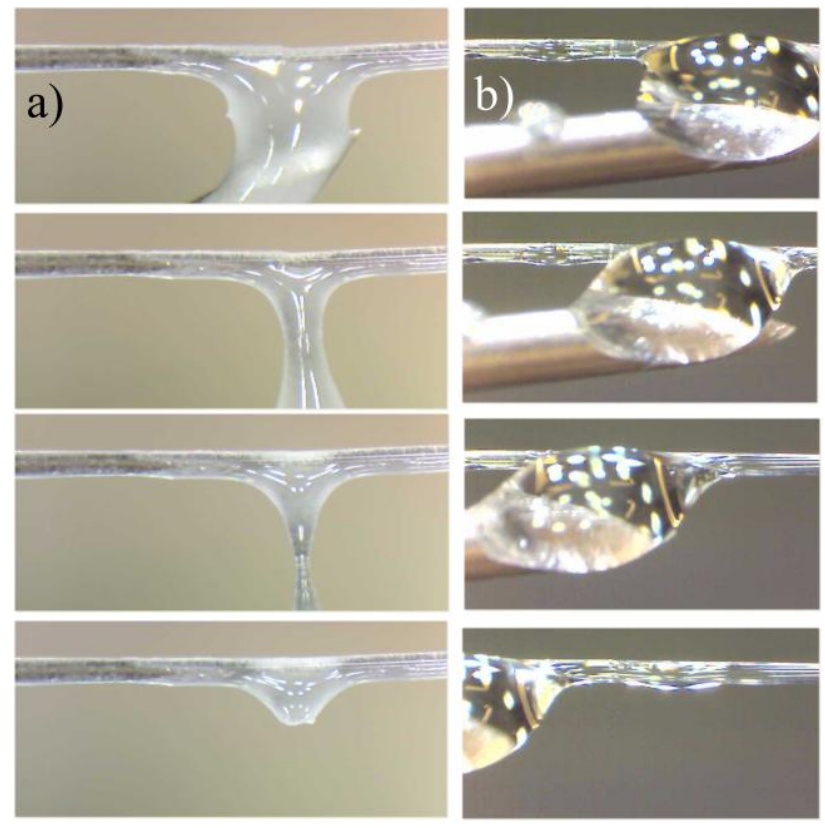

Fig. 4. Precise application of glue with a needle: a) needle slowly moved away; b) needle slowly moved along the connected fibers. 

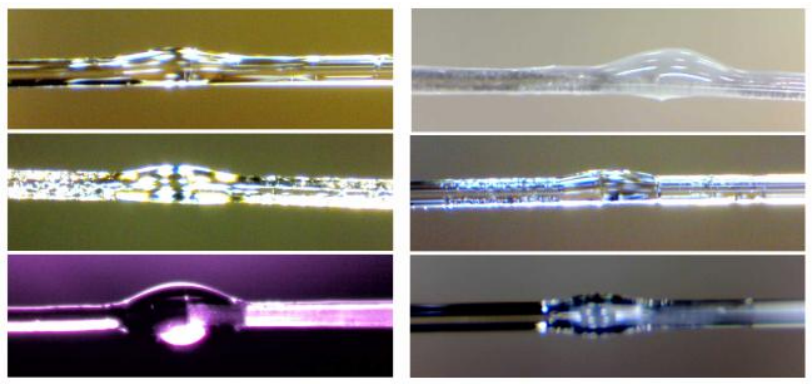

Fig. 5. Examples of the bonds created with a needle applicator, the violet picture was taken during irradiation with UV light. All presented bonds were mechanically stable when removed from the setup (although sensitivity to bending was sometimes noticed).

We have also briefly tested a second method of precise glue application based on tapered micro-capillaries (Fig. 6a). We decided to taper the capillary to obtain the smallest possible droplet of the glue. If the one end of a capillary was filled with some amount of glue, it was possible to pull it away pneumatically by using a hermetic syringe connected to the second end of the capillary. However, such control was not precise because the response of an applicator depended on the viscosity of the glue and the amount of liquid in the capillary. Moreover, the response to the syringe movement was delayed so it was hard to obtain repeatable micro-droplets. Recently, we are testing a slightly modified approach in which the movement of a syringe plunger is controlled with a micrometer (Fig. 6c). We expect that such a microinjector would allow for precise control of the volume of the micro-drop formed at the output of the capillary.
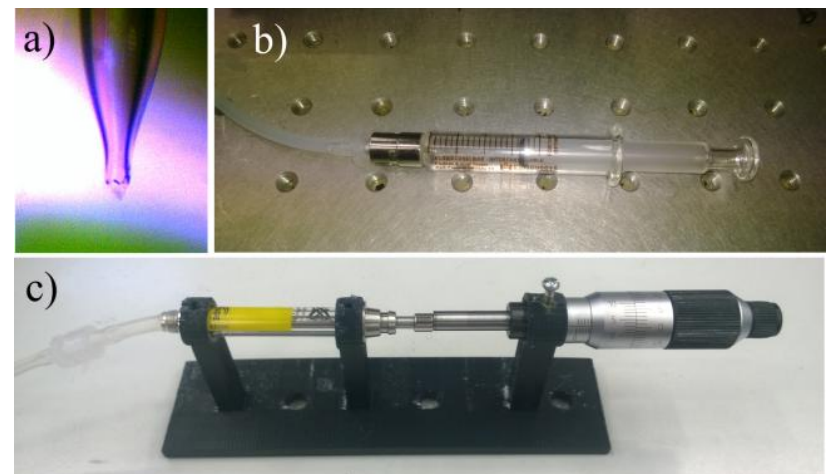

Fig. 6. Idea of the adhesive application with a micro-capillary a) example of the tapered capillary; b) hermetic syringe used for pneumatic application of glue; c) prototype of the home made micro-injector.

Currently, the most repeatable results could be obtained by using the applicator based on a large medical needle and in most cases such an application would be sufficient. However, if the smallest possible bond is required, the micro-injector method could be the only suitable solution.

The losses of the obtained connections depended mainly on the modal properties of connected fibers. When two identical fibers (i.e. two SM fibers) were connected, the losses were typically lower than $0.5 \mathrm{~dB}$. Interesting results are possible if two identical fibers with polymer coating are connected: a very strong, small and practically bending-insensitive connections are possible (Fig.7).
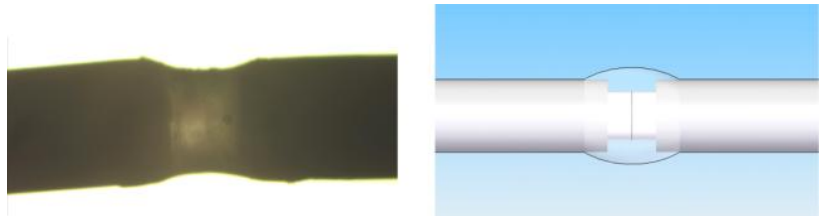

Fig. 7. Low-loss $(<0.2 \mathrm{~dB})$ connections between two SM fibers are possible. If the polymer coating is removed for $\sim 0.25 \mathrm{~mm}$ at both fibers, the obtained connections are very strong and practically not sensitive to bending (tested with a bend radius as small as $2 \mathrm{~cm}$ ).

Of course, photochemical bending is well suitable for connecting fibers made of different materials. In particular, we have tested a broad range of connections between classic SM fibers (made of silica) with microstructured optical fibers (including those made of multi-component glasses) as well as liquid crystal filled fibers. The losses were typically from $1 \mathrm{~dB}$ to few $\mathrm{dBs}$ (mode missmatch depening).

The presented method is very promising, in particular, that the whole process could be relatively easy automated. The automated setup with 5-axis fiber alignment, 3-axis positioning of the glue applicator controlled with a microinjector and with two cameras with an adjustable focus would require simultaneous control of 11 actuators.

This work was supported by the National Centre for Research and Development as part of the project LIDER/05/208/L3-11/NCiBR/2012.

\section{References}

[1] J. Ballato, A.F. Abouraddy, Opt. Mat. Expr. 2, 1680 (2012).

[2] I. Hatakeyama, H. Tsuchiya, IEEE J. Quant. Electr. 14, 614 (1978).

[3] K. Inada, O. Watanabe, H. Taya, IEEE J. Select. Areas in Comm. 4, 706 (1986).

[4] L. Xiao, M.S. Demokan, W. Jin, Y. Wang, C. Zhao, J. Lightwave Technol. 25, 3563 (2007).

[5] R. Al-Mahrous, R. Caspary, W. Kowalsky, J. Lightwave Technol. 32, 302 (2014).

[6] S. Ertman, A.H. Rodríguez, M. M. Tefelska, M. S. Chychłowski, D. Pysz, R. Buczyński, E. Nowinowski-Kruszelnicki, R. Dąbrowski, T.R. Woliński, J. Lightwave Technol. 30, 1208 (2012).

[7] M.M. Kozak, W. Kowalsky, R. Caspary, Electron. Lett. 41, 21 (2005).

[8] R. Al-Mahrous, R. Caspary, W. Kowalsky, J. Lightwave Technol. 32, 1669 (2014). 